\title{
The Signal Detection and Control Circuit Design for Confocal Auto-Focus System
}

\author{
Liu Yin ${ }^{1}$, Yu Jin ${ }^{1}$, Mo Zeqiang ${ }^{1}$ \\ ${ }^{1}$ Academy of Opto-Electronics, Chinese Academy of Sciences, Dengzhuang South road, Haidian District, 100094, Beijing, China
}

\begin{abstract}
Based on the demands of Confocal Auto-Focus system, the implementation method of signal measurement circuit and control circuit is given. Using the high performance instrumental amplifier AD620BN, low noise precision FET Op amplifier AD795JRZ and ultralow offset voltage Op amplifier OP07EP, a signal measurement circuit used to converse the two differential light intensity signal to electric signal is designed. And a control circuit which takes MCU MSP430F149 as core processes the former signal and generate a control signal driving the platform for autofocusing. The experimental results proved the feasibility and correctness of circuits. And the system meets the design requirement.
\end{abstract}

Keywords: Confocal Auto-Focus; Photoelectric Conversion; Data Acquisition; MSP430F149

\section{Introduction}

Confocal auto-focus system is used for microscopic image acquisition device, which is sensitive to the focal length. Compared to conventional auto-focus methods such as ranging method, focus detection method and image processing method, the confocal auto-focus method can effectively suppress the effects of noise and temperature drift, having the advantages in accuracy(up to nano-scale), less computational complexity and high speed[1-2].

The system consists of a light source, a measured object, a focusing lens, two pinhole aperture and two photodetectors. In working a laser with the wavelength of $632.8 \mathrm{~nm}$ goes through the focusing lens and then irradiate the target object. The reflected light respectively goes through the pinhole aperture and into the two photodetectors. According to the principle of confocal the intensity difference of two detected lights can reflect the direction and value of the defocus distance.

The confocal optical signal detected by the system has a high sensitivity and linearity requirement, but not caring much about dynamic characteristic. Taking into account the high-frequency electromagnetic interference generated by the surrounding environment, it is need to design a low-noise, high SNR, and high CMRR photoelectric signal detection circuit. In this paper, a signal detection circuit based on the high performance instrumental amplifier AD620BN is capable of conversing the confocal optical signals to electrical signal. Via signal conditioning, differential amplifier and lowpass filter, the signal is send into the control circuit of MCU MSP430F149 for processing, generating focusing control signal to drive the electro-platform to complete auto-focusing process

\section{Photoelectric Signal Detection Circuit}

\subsection{Principle of Confocal Photoelectric Signal Detection}

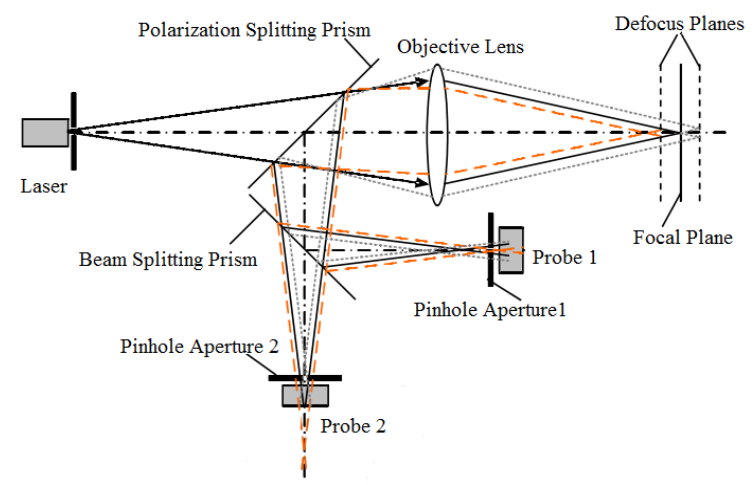

Fig. 1 Optical Principle of Confocal System

As shown in Fig. 1, the two pinhole apertures are located in the conjugate planes of the two defocus planes which are at the bilateral symmetric positions of the focal plane. The two reflected lights which are split equally by the Beam Splitting Prism pass through the pinholes and irradiate at the two probs. When the object is at the focal plane the two detectors receive the lights with equal intensity, but when there is a distance between the object and the focal plane, the intensity of two lights are different. The received light intensity of two probe and their difference curve are shown in Fig. 2. The difference 
at the point of origin is corresponding to the focal position. So the symbol of the difference can reflect the direction of defocus position and the value of difference can used to calculate the length of the defocus distance.

The introduction of differential optical path can effectively suppress the noise and the temperature drift on the measurement and thereby improves system SNR[2].

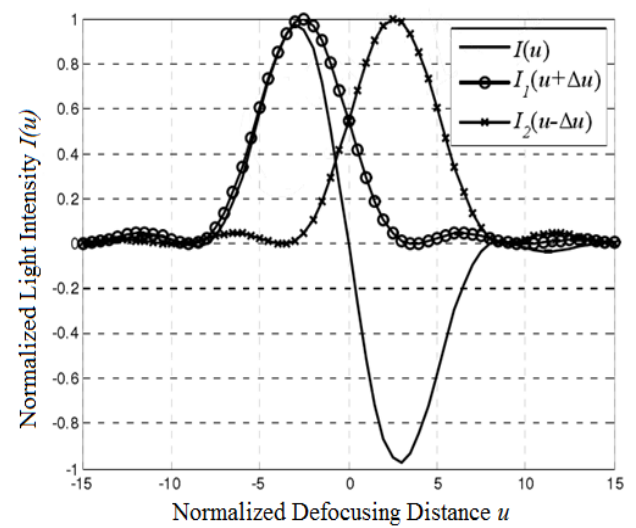

Fig. 2 Confocal Light Response Curve

The photoelectric signal detection circuit is consist of the pre-circuit for photoelectric conversion, the signal conditioning and differential circuit, Second-Order LowPass Filter and pseudo-zero elimination circuit. The circuit structure diagram is shown in Fig. 3 and the specific details are described below.

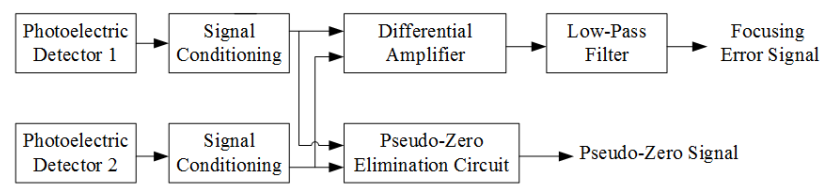

Fig. 3 Structure diagram of photoelectric signal detection circuit

\subsection{Circuit Design}

Using photodiode, the light intensity can be conversed into weak current signal $(\mu \mathrm{A})$. Considering the high demand of linearity and the general demand of dynamic characteristics, the photodiode is working on the zero bias mode instead of the reverse bias mode. The former one has higher linearity and can avoid the latter one's Dark Current when there is no light at all[3-4].

The output voltage Uo is determined in Equation 1 when taking into account the OP amplifier's Offset Voltage UIO and Offset Current IIO. The R represents feedback resistance, $\mathrm{Rd}$ is photodiode junction resistance, I is the weak current signal generated by photodiode and Isc is short-circuit current of photodiode.

$$
U_{0}=U_{10} \times\left(1+R / R_{d}\right)+R \times\left(I+I_{10}+I_{s C}\right)
$$

It's obvious that the output voltage is effected by the Offset Current and the Offset voltage. In order to minimize the error the low noise precision FET Op amplifier AD795JRZ is used for pre amplifier, which input offset voltage is up to $500 \mu \mathrm{V}$ at $25^{\circ} \mathrm{C}$, and its maximum offset current is $1 \mathrm{pA}$. To improve the driving capability of the amplifier circuit while avoiding the adverse effects of the subsequent circuit, a voltage follower made of OP07EP is added after the detection circuit.

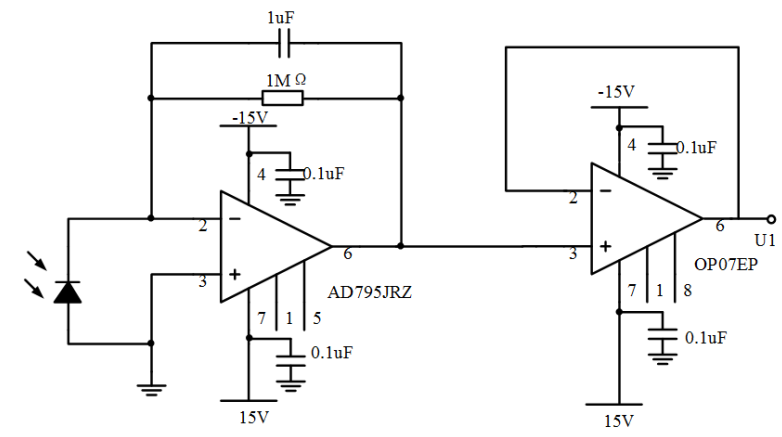

Fig. 4 Structure diagram of photoelectric signal detection circuit

The voltage generated by the pre-circuit is still weak, so a post-stage voltage amplifier is needed to amplify the signal to an appropriate range.

Due to the different sensitivity of photodiodes as well as the circuit distribution parameters, the circuit use the rheostat as a feedback resister. Equation 2 shows that by adjusting the resistance R2 can make the two ways of detection circuit gain the same.

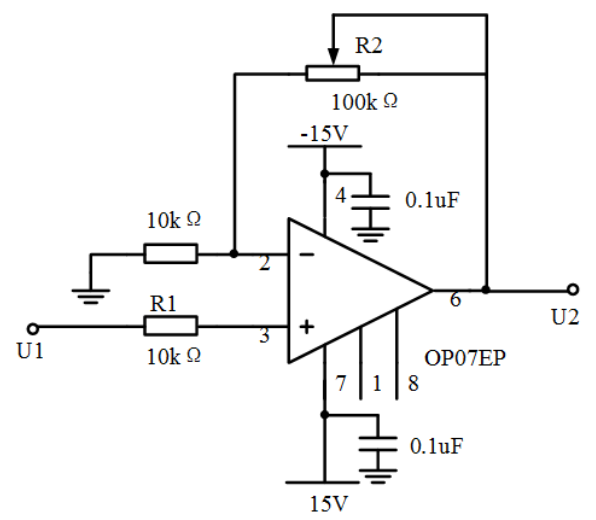

Fig. 5 Signal Conditioning Circuit

$$
U_{2}=U_{1} \times\left(1+R_{2} / R_{1}\right)
$$

The differential circuit is used to calculate the difference between the two detected voltages and generated the error voltage. In order to reduce common mode interference, high performance instrumental amplifier AD620BN is used to make the differential circuit. This amplifier's offset voltage is up to $50 \mu \mathrm{V}$ and offset current is $1.0 \mathrm{nA}$. To minimize the gain error, the gain is set to 1 when the gain error is up to $0.02 \%$. The equation of this circuit is Equation. 3.

$$
U_{01}=1 \times\left(U_{i 1}-U_{i 2}\right)
$$




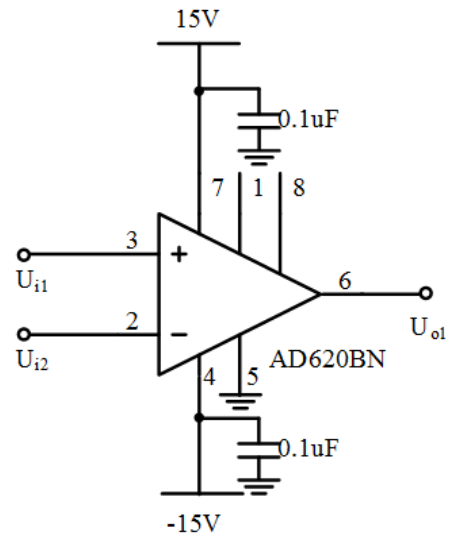

Fig. 6 Differential amplifier Circuit

A low-pass filter is necessary because the surrounding environment's high-frequency electromagnetic interference and the indoor lighting stroboflash will cause noise. Common low-pass filters are Butterworth, Chebyshev, and Bessel ect. The Chebyshev filter has the advantages like equal ripple voltage in passband, high cutoff characteristics and steep attenuation slope. The error between its frequency response curve and the ideal ones is the least[5]. So in this paper the Chebyshev is chosen as the low-pass filter with $30 \mathrm{~Hz}$ cutoff frequency. The transfer function is Equation 4 and the specific parameters are shown in Fig. 7.

$$
G(s)=\frac{A_{u p}(s)}{1+\left[3-A_{u p}(S)\right] s R C+(\operatorname{sRC})^{2}}
$$

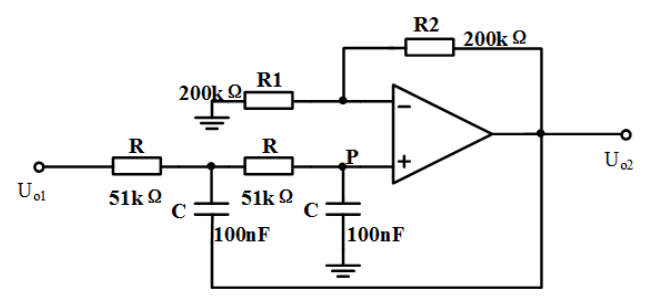

Fig. 7 Second-Order Chebyshev Low-Pass Filter Circuit

To ensure its frequency response characteristic, use the Multisim 13 to simulate the circuit and the result is shown in Fig. 8.

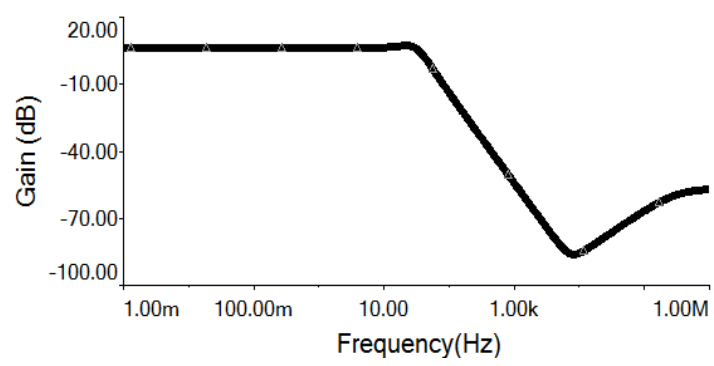

Fig. 8 Multisim Simulation Curve of Second-Order Chebyshev

In actual condition, when the object is out of the valid focus range, the differential signal well also be approximately zero. In order to avoid this situation, a pseudo-zero elimination circuit is introduced. Only when the sum of two detected voltage signal is higher than the threshold voltage set by the MCU, the system will work on the normal mode.

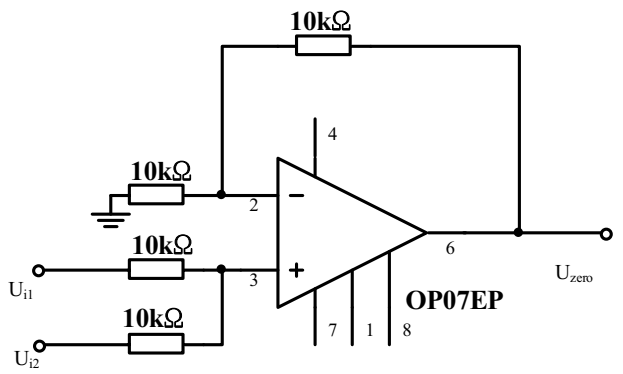

Fig. 9 Pseudo-Zero Elimination Circuit

\section{Signal acquisition and control circuit}

This part is based on the MCU MSP430F149. The MCU acquires the focusing error signal and then generates a control signal to drive the high accuracy electric-platform to complete focus. A multi-channel ADC and a comparator are put inside the $\mathrm{MCU}$ which will greatly simplify the external circuit design work. When the system works, the pseudo-zero signal will compare with the internal reference voltage by the inner comparator of $\mathrm{MCU}$, if the result is high level the focusing error signal will converse into digital signal. The positive reference $\mathrm{VR}+$ is power voltage $(3.3 \mathrm{~V})$ and the negative $\mathrm{VR}$ - is GND $(0 \mathrm{~V})$. The conversion function is Equation 5. To reduce errors, the MCU will continuous sample the signal 32 times and calculate the average. The program flow chart is shown in Fig. 10.

$$
N_{A D C}=\frac{4095 \times\left(V_{R N}-V_{R-}\right)}{\left(V_{R}-V_{R-}\right)}
$$
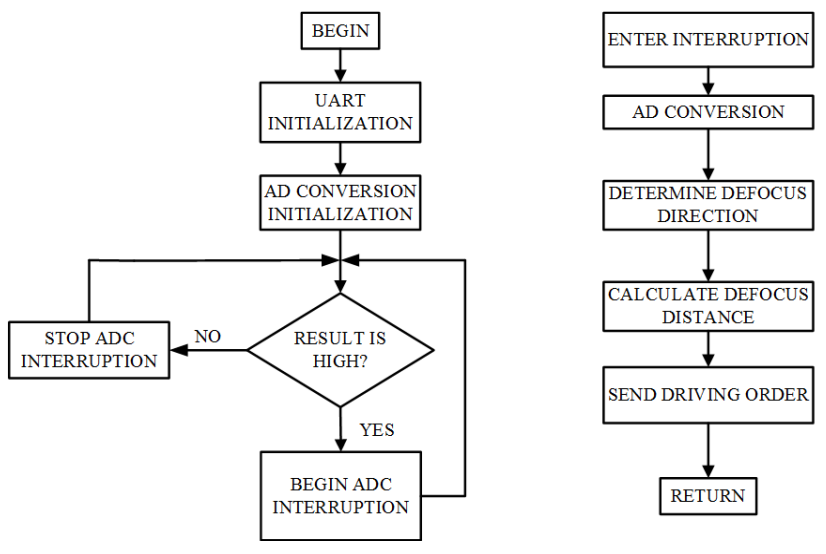

Fig. 10 Program Flow Chart

\section{Experiment Results}

Moving the electric-platform on the step size of $10 \mu \mathrm{m}$, record the focusing error voltage 5 times. The result voltage scatter diagram and the fitting result is shown in Fig. 11. Using $\mathrm{x}$ represent object position $(\mu \mathrm{m})$ and $\mathrm{y}$ 
represent voltage $(\mathrm{V})$, the linear function is (6). The system sensitivity is $187.9 \mathrm{mV} / \mu \mathrm{m}$.

$$
y=0.1879 x+0.4724
$$

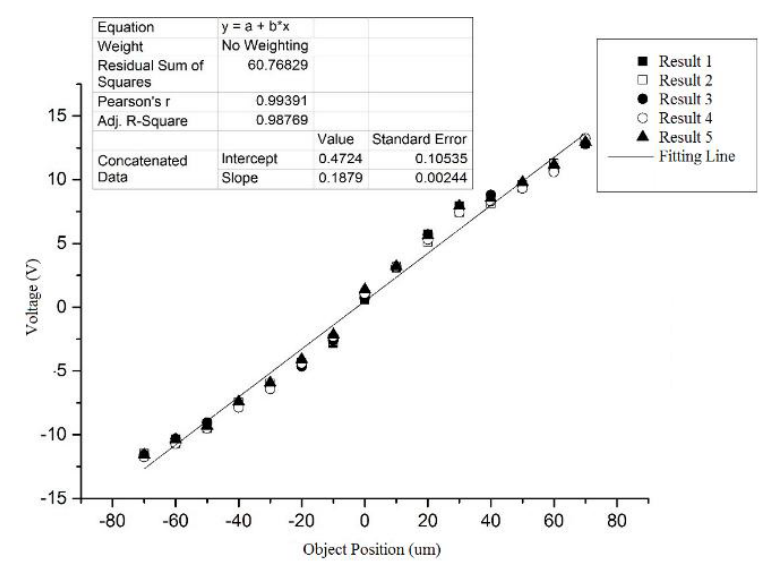

Fig. 11 Voltage Scatter Diagram and Fitting Result

In the accuracy test, at the step size of $20 \mu \mathrm{m}$, change the object position manually. After the system is stable, record the differential voltage. Using (1.5), it's easy to calculate the position and determine whether the system is compliance with the design index. To avoid the return error of the platform, movement direction of each test must be same. So in the Table 1.1 there are two groups of results with different directions. The extremums among the results are $-197 \mathrm{mV}$ and $209 \mathrm{mV}$, which represent defocusing distance $-1.05 \mu \mathrm{m}$ and $1.11 \mu \mathrm{m}$.

Table 1 Accuracy test results

\begin{tabular}{|c|c|c|c|c|c|c|}
\hline \multirow{2}{*}{$\begin{array}{c}\text { Distance } \\
(\boldsymbol{\mu m})\end{array}$} & \multicolumn{3}{|c|}{$\begin{array}{c}\text { Positive Movement } \\
\text { Differential Voltage(mV) }\end{array}$} & \multicolumn{3}{c|}{$\begin{array}{c}\text { Negative Movement } \\
\text { Differential Voltage(mV) }\end{array}$} \\
\cline { 2 - 7 } & $\boldsymbol{V 1}$ & $\boldsymbol{V 2}$ & $\boldsymbol{V 3}$ & $\boldsymbol{V 1}$ & $\boldsymbol{V} 2$ & $\boldsymbol{V} 3$ \\
\hline 0 & -55 & 53 & 60 & -34 & 84 & 23 \\
\hline 20 & -72 & 84 & -48 & 209 & 76 & 127 \\
\hline 40 & 138 & -79 & 137 & 74 & -21 & -61 \\
\hline 60 & -124 & 134 & 126 & 120 & 34 & 26 \\
\hline 80 & -154 & -83 & 37 & -187 & 42 & 83 \\
\hline 100 & 202 & 162 & -197 & 126 & 48 & -71 \\
\hline
\end{tabular}

\section{Conclusion}

Confocal auto-focus system can achieve the auto-focus control of microscopic image acquisition. This system has the advantages of low noise, high accuracy, low computational complexity and high speed of control. This paper designed the photoelectric signal detection circuit as well as signal acquisition and control circuit. The program process of MCU is also presented. The detection circuit changes the light signal into analog voltage signal, the MCU acquire the analog signal and by signal processing produces the available moving order to control the electric-platform. The linearity experiment results prove the feasibility and the correctness of the detection circuit. And the accuracy test shows that the system's focusing accuracy fits the design order which is $\pm 50 \mu \mathrm{m}$.

\section{Acknowledgement}

This work is supported by Cross-disciplinary Collaborative Teams Program for Science, Technology and Innovation, Chinese Academy of Sciences.

\section{References}

1. Chau-Hwang Lee, Chin-Lin Guo, Jyhpyng Wang. Optical measurement of the viscoelastic and biochemical responses of living cells to mechanical perturbation. Optics letters, 1998, 23(4):307-309.

2. Wang Y H. Research on the Detecting Technologies and System Based on Whole-Field Parallel Confocal. Hefei: Hefei University of Technology, 2004.

3. Li Y M, Chen W T. Circuit Design of Weak Optical Signal Preamplifier. Electronic Component \& Device Applications, 2007, 9(8):51-53.

4. Liu R L, Yin D K. Design of a Photoeletric Detecting Circuit for Laser Interferometer. Semiconductor Optoelectronics, 2010, 31(2):284-287.

5. Tong S B, Hua C Y. Basic Analog Electronic Technology. Beijing: Higher Education Press, 2006.

6. Zhu C L, Multism Electronic Circuit Simulation Tutorial. Xian: Xidian University Press, 2007. 